\title{
Population pharmacokinetics and pharmaco- dynamics of bivalirudin in young healthy Chinese volunteers
}

\author{
Dong-mei ZHANG ${ }^{1, \#}$, Kun WANG ${ }^{2, \#}$, Xia ZHAO ${ }^{1}$, Yun-fei $\mathrm{LI}^{2}$, Qing-shan ZHENG ${ }^{2, *}$, Zi-ning WANG ${ }^{1}$, Yi-min CUI $^{1, *}$ \\ ${ }^{1}$ Department of Pharmacy, Peking University First Hospital, Beijing 100034, China; ${ }^{2}$ Laboratory of Pharmacometrics, Center for Drug \\ Clinical Research, Shanghai University of Traditional Chinese Medicine, Shanghai 201203, China
}

\begin{abstract}
Aim: To investigate the population pharmacokinetics (PK) and pharmacodynamics (PD) of bivalirudin, a synthetic bivalent direct thrombin inhibitor, in young healthy Chinese subjects.

Methods: Thirty-six young healthy volunteers were randomly assigned into 4 groups received bivalirudin $0.5 \mathrm{mg} / \mathrm{kg}, 0.75 \mathrm{mg} / \mathrm{kg}$, and $1.05 \mathrm{mg} / \mathrm{kg}$ intravenous bolus, $0.75 \mathrm{mg} / \mathrm{kg}$ intravenous bolus followed by $1.75 \mathrm{mg} / \mathrm{kg}$ intravenous infusion per hour for $4 \mathrm{~h}$. Blood samples were collected to measure bivalirudin plasma concentration and activated clotting time (ACT). Population PK-PD analysis was performed using the nonlinear mixed-effects model software NONMEM. The final models were validated with bootstrap and predictioncorrected visual predictive check (pcVPC) approaches.

Results: The final PK model was a two-compartment model without covariates. The typical PK population values of clearance (CL), apparent distribution volume of the central-compartment $\left(V_{1}\right)$, inter-compartmental clearance $(\mathrm{Q})$ and apparent distribution volume of the peripheral compartment $\left(V_{2}\right)$ were $0.323 \mathrm{~L} \cdot \mathrm{h}^{-1} \cdot \mathrm{kg}^{-1}, 0.086 \mathrm{~L} / \mathrm{kg}, 0.0957 \mathrm{~L} \cdot \mathrm{h}^{-1} \cdot \mathrm{kg}^{-1}$, and $0.0554 \mathrm{~L} / \mathrm{kg}$, respectively. The inter-individual variabilities of these parameters were $14.8 \%, 24.2 \%$, fixed to $0 \%$ and $15.6 \%$, respectively. The final PK-PD model was a sigmoid $E_{\text {max }}$ model without the Hill coefficient. In this model, a covariate, red blood cell count (RBC*), had a significant effect on the $\mathrm{EC}_{50}$ value. The typical PD population values of maximum effect $\left(E_{\max }\right), \mathrm{EC}_{50}$, baseline ACT value $\left(E_{0}\right)$ and the coefficient of RBC* on $\mathrm{EC}_{50}$ were $318 \mathrm{~s}, 2.44 \mathrm{mg} / \mathrm{L}, 134 \mathrm{~s}$ and 1.70 , respectively. The inter-individual variabilities of $E_{\max }, \mathrm{EC}_{50}$, and $E_{0}$ were $6.80 \%, 46.4 \%$, and $4.10 \%$, respectively.

Conclusion: Population PK-PD models of bivalirudin in healthy young Chinese subjects have been developed, which may provide a reference for future use of bivalirudin in China.
\end{abstract}

Keywords: anticoagulation therapy; bivalirudin; activated clotting time; healthy young Chinese subjects; population pharmacokinetics and pharmacodynamics

Acta Pharmacologica Sinica (2012) 33: 1387-1394; doi: 10.1038/aps.2012.37; published online 4 Jun 2012

\section{Introduction}

Anticoagulation therapy during cardiac percutaneous and surgical interventions is at the forefront of interventional cardiovascular medicine today. Traditionally, unfractionated heparin (UFH) and low molecular-weight heparin (LMWHs) have been utilised as the primary anticoagulant during cardiac surgery. However, both UFH and LMWHs have inherent limitations, often with potentially deleterious clinical consequences, such as heparin-induced thrombocytopenia (HIT).

\footnotetext{
\#These two authors contributed equally to this work.

* To whom correspondence should be addressed.

E-mail cuiymzy@126.com (Yi-min CUI); zhengqscn@21cn.com (Qing-shan ZHENG)

Received 2012-02-06 Accepted 2012-03-23
}

Additionally, hemodynamic instability after protamine administration to reverse UFH during cardiopulmonary bypass (CPB) has been reported to be associated with a $13.2 \%$ posthospital admission mortality rate ${ }^{[1]}$. These untoward sequelae have compelled both basic science and clinical investigators to search for alternate anticoagulation strategies.

Bivalirudin, a synthetic bivalent direct thrombin inhibitor (DTI), has its history rooted with that of hirudin, a natural irreversible bivalent DTI derived from the salivary glands of the medicinal leech, Hirudo medicinalis ${ }^{[2]}$. Bivalirudin was previously known as Hirulog ${ }^{\mathrm{TM}}$ and was developed by Biogen as a synthetic form of hirudin that incorporates the binding affinity to both the catalytic active site and the anion binding exosite of thrombin ${ }^{[3]}$. Currently, bivalirudin is manufactured by Medicines Company (Parsippany, NJ, USA) at a cost of 
approximately US\$325 for a $250-\mathrm{mg} \mathrm{vial}^{[4]}$.

Extensive investigations have confirmed that bivalirudin has several therapeutic features superior to UFH and LMWHs, including more predictable pharmacokinetics (PK) and pharmacodynamics (PD) behaviour, a lower proclivity to generate an immune response, and an equivalent suppression of acute ischemic events with fewer bleeding complications ${ }^{[5-8]}$.

The FDA approved bivalirudin in 2000 under the brand name Angiomax, and the Committee for Medical Products for Human Use (CHMP) approved it in 2004 under the name Angiox; however, the drug has not been marketed in China until now. During the last several decades, there has been an increase in both the economic growth and the aging population in China. Along with this, the morbidity and mortality of acute coronary syndrome (ACS) has greatly increased, and percutaneous coronary intervention (PCI) treatment has also become more popular. Due to these situations and the fact that bivalirudin is highly recommended for anticoagulation therapy, it should be considered for use in China. Therefore, it is currently at an advanced development stage in China, with several ongoing clinical trials at this time.

In general, it is important to carefully explore drug properties at the early stages of drug development. In 1972, Sheiner et al proposed using nonlinear mixed-effects modelling to estimate population PK parameters. Although originally described in the context of therapeutic drug monitoring, the population approach has been most extensively applied to clinical trials and drug development over the past 30 years ${ }^{[9]}$. Population PK-PD models are a key component of modelbased drug development ${ }^{[10-12]}$. Performing population PK-PD modelling using nonlinear mixed-effects modelling allows, apart from the estimation of fixed effects (the PK and PD model estimates), the quantification of random effects as within- and between-subject variability, which is critically valuable in the drug development process.

The purpose of this work was to identify and estimate characteristic parameters of a population PK-PD model for bivalirudin in healthy young Chinese subjects. This was done to achieve a better understanding of the pharmacological properties of bivalirudin and to provide more information for the design of later clinical trials of bivalirudin.

\section{Materials and methods}

\section{Study design and drug analysis}

All patients gave written informed consent, and the Independent Ethics Committee and the Institutional Review Board of Peking University First Hospital approved the study. Briefly, 48 healthy Chinese Han ethnic subjects were enrolled in the study and randomly assigned to either the bivalirudin group $(n=36)$ or placebo treatment group $(n=12)$, following simple randomisation procedures (computerized random number). The placebo group data were not used in the population PK-PD modelling; however, these data were of great value for evaluating the accuracy of PD determinations and distinguishing the bivalirudin anticoagulant effect from the placebo effect. The bivalirudin treatment group consisted of four dose levels:
$0.5 \mathrm{mg} / \mathrm{kg}, 0.75 \mathrm{mg} / \mathrm{kg}$, and $1.05 \mathrm{mg} / \mathrm{kg}$ intravenous bolus, and $0.75 \mathrm{mg} / \mathrm{kg}$ intravenous bolus followed by an infusion of $1.75 \mathrm{mg} / \mathrm{kg}$ per hour for $4 \mathrm{~h}$ ( $n=9$ for each dose). The selection of doses was based on the instructions for bivalirudin use marketed in the United States. The group that received the intravenous bolus of bivalirudin followed by an infusion of the drug received the same dose as that used clinically abroad. Intensive pharmacokinetic and pharmacodynamic sampling was performed. Bivalirudin plasma concentrations were determined using validated liquid chromatography-tandem mass spectrometry. Activated clotting time (ACT) measurement was performed using the Hemochron Response device (International Technidyne Corp, New Albany, IN, USA). Details of the study design, pharmacokinetic and pharmacodynamic sampling, determination of bivalirudin concentration and ACT were described previously ${ }^{[13]}$.

\section{Population pharmacokinetic modelling}

Plasma concentration-time data for bivalirudin were modelled using a nonlinear mixed-effects approach with NONMEM (version 7.2, ICON Development Solutions, USA), with the aid of Wing for NONMEM and Perl speak to NONMEM (PsN), and Xpose 4.3.2, an S-Plus based population analysis aid with data set checking, exploration, visualisation, model diagnostics, candidate covariate identification and model comparison. All parameters were estimated using the First-Order Conditional Estimation method (FOCE) with eta-epsilon Interaction.

\section{Basic model selection}

Based on the bivalirudin concentration-versus-time curves from the healthy subjects, one- and two-compartment models were chosen. The data were fit without introducing any covariates. The objective function value (OFV), data fitness, and parameter rationality were comprehensively compared to choose the best base model.

\section{Random effect model}

The random effects of population pharmacokinetics included inter-individual variability and residual error.

A lognormal distribution of parameters was assumed for inter-individual variability. Therefore, the distribution of the PK parameters was defined as

$$
P_{\mathrm{i}}=\theta_{P} \cdot \operatorname{Exp}\left(\eta_{P_{\mathrm{i}}}\right)
$$

where $P_{\mathrm{i}}$ is the individual estimate for a model parameter $P$, such as CL in the ith individual, $\theta_{P}$ is the population median of the parameter $P$, and $\eta_{P \mathrm{i}}$ describes the ith individual's deviation from the population mean estimated as a distribution, assuming a zero mean and a variance of $\omega$.

The residual error of the model assumed a normally distributed random variable with a mean of zero and a variance of $\sigma^{2}$. Additive, proportional and proportional plus additive models were tested.

\section{Covariate model}

After the identification of the base, or covariate-free model, scatter plots of individual PK parameters versus available 
demographic and biological characteristics were generated. In addition, stepwise covariate modelling (SCM) by PsN was used to initially screen for potential, significant covariates. The tested covariate models included additive, proportional and power models.

The potential covariates that were screened by SCM were individually introduced into the basic model and screened again with NONMEM. In NONMEM, all covariates were investigated by forward inclusions and backward deletion techniques. Each selected covariate was first tested by a univariate NONMEM analysis to confirm its relevance. A decrease in OFV of at least 3.84 units, corresponding to $P<0.05$, was first required to consider the covariate in the intermediate model. In a second step, all significant covariates were added simultaneously to the intermediate model, and then, each covariate was independently removed from the full model to confirm its relevance. This is known as the backward deletion strategy, and the resulting variation in OFV as compared to the full model was examined. An increase in OFV of $>6.64$ $(P<0.01)$ was required to confirm the significance of the covariate and to take it into account in the final model. A more conservative criterion was used for model reduction $(P<0.01)$ than for model building $(P<0.05)$ to reduce the likelihood of accepting a false-positive covariate.

\section{Model validation}

The accuracy and robustness of the final model were evaluated using the resampling techniques of the bootstrap method and prediction-corrected visual predictive check ( $\mathrm{pcVPC}$ ).

The bootstrap method involves repeated random sampling of subjects in the database. The original dataset is replaced to produce another dataset of the same size, but with a different combination of subjects. Resampling was repeated 1000 times. The median values and $95 \%$ confidence intervals for the parameters estimated from the bootstrap sets that produced successful minimisation and convergence were compared to the parameter estimates obtained from the original data set.

Visual predictive check (VPC) is a valuable method for checking model performance. However, the shortcomings of the traditional VPC have been highlighted before ${ }^{[14]}$; therefore, pcVPC offers a solution to these problems while retaining the visual interpretation of the traditional $\mathrm{VPC}^{[15]}$. By using the pcVPC, both the observations and model predictions were normalised for the typical model predictions. One thousand data sets were simulated from the final model parameter estimates, and the non-parametric $95 \%$ confidence intervals for the median, 5th and 95th percentiles based on the 1000 simulated datasets were calculated and represented together for visual inspection.

\section{Population pharmacokinetic/pharmacodynamic modelling}

In developing the population PK-PD models, the IPP (Individual PK Parameters) sequential approach ${ }^{[16]}$ was used. The population PK model was built first as mentioned above, and then the estimated individual PK parameters were fixed to individual estimations from the final PK model for estimating the PD parameters.

Bivalirudin is a direct thrombin inhibitor and is administered as an intravenous injection. Additionally, it is not a prodrug, meaning it is the active form only and does not need to be metabolised in vivo to exhibit anticoagulation activity. Considering these characteristics of bivalirudin, and the fact that thrombin is the last enzyme in the clotting cascade, the direct response PD model was chosen.

The sigmoid $E_{\max }$ effect model with or without sigmoidicity (parameter $\gamma$ ) and without time delay was selected as the base PD model. The $E_{\max }$ model was described by the following equation:

$$
E=E_{0}+\left[\left(E_{\max }-E_{0}\right)^{*} C_{\mathrm{e}}{ }^{\gamma}\right] /\left(C_{\mathrm{e}}{ }^{\gamma}+E C_{50}{ }^{\gamma}\right)
$$

where $E$ indicates the effect of bivalirudin on ACT; $E_{0}$ is the baseline ACT value; $E_{\max }$ is the maximum effect; $C_{\mathrm{e}}$ is the bivalirudin concentration of the central compartment, which was also the effect compartment; $\gamma$ is the sigmoidicity (Hill) factor; and $\mathrm{EC}_{50}$ is the bivalirudin concentration at $50 \%$ maximum effect.

The process of building the random effect model and covariate model, and the validation process of the final model, were the same as the procedures performed for the population PK analysis.

\section{Results}

\section{Volunteer characteristics}

The demographic and biological characteristics of the 36 healthy volunteers are summarised in Table 1 .

\section{Population pharmacokinetic modelling}

A total of 423 plasma bivalirudin measurements were taken and made available for population PK modelling.

\section{Model building}

We compared the OFV of the one- and two-compartment models and found that the OFV value of the two-compartment model decreased by $1129.144(P<0.0001)$ compared to the onecompartment model. Additionally, the fit of the two-compartment model indicated that the population predictive value (PRED), individual predictive value (IPRED), and observed value had better correlations, suggesting that the twocompartment model was preferable to the one-compartment model. Therefore, the two-compartment model was chosen as the basic model for the next population pharmacokinetic analysis.

The exponential model was chosen as the inter-individual variability model. A proportional error model was shown to be sufficient for the characterisation of the residual unexplained variability (RUV). Thirty covariates, including demographic factors and laboratory indicators, were analysed in the present study. No covariate showed significant influence on the pharmacokinetic parameters.

Therefore, the final population PK model of bivalirudin in healthy Chinese subjects was a two-compartment, covariatefree, inter-individual variability exponential model, with residual error described by a proportional error model. 
Table 1. Demographic and biological characteristics of the 36 young, healthy volunteers.

\begin{tabular}{lcccc}
\hline Characteristics & $\begin{array}{c}0.5 \mathrm{mg} / \mathrm{kg} \\
\text { Intravenous bolus } \\
(n=9)\end{array}$ & $\begin{array}{c}0.75 \mathrm{mg} / \mathrm{kg} \\
\text { Intravenous bolus } \\
(n=9)\end{array}$ & $\begin{array}{c}1.05 \mathrm{mg} / \mathrm{kg} \\
\text { Intravenous bolus } \\
(n=9)\end{array}$ & $\begin{array}{c}0.75 \mathrm{mg} / \mathrm{kg} \text { Intravenous bolus } \\
\text { followed by } 1.75 \mathrm{mg} / \mathrm{kg} \text { per } \\
\text { hour infusion for } 4 \mathrm{~h}(n=9)\end{array}$ \\
\hline Gender (males/females; $n)$ & $4 / 5$ & $3 / 6$ & $7 / 2$ & $3 / 6$ \\
Age $($ year $)$ & $34.2(32-37)$ & $33.7(31-37)$ & $33.0(29-36)$ & $33.1(29-37)$ \\
Weight $(\mathrm{kg})$ & $61.6 \pm 9.1(50.5-78)$ & $61.5 \pm 6.7(57-74)$ & $60.7 \pm 7.3(50-70)$ & $55.4 \pm 6.5(50-70)$ \\
$\mathrm{BMI}\left(\mathrm{kg} / \mathrm{m}^{2}\right)$ & $22.8 \pm 2.0(19.3-24.9)$ & $23.4 \pm 1.1(21.5-24.4)$ & $22.1 \pm 1.7(19.1-24.5)$ & $22.0 \pm 0.9(20.6-23.2)$ \\
$\mathrm{PLT}\left(\times 10^{9} / \mathrm{L}\right)$ & $203.9 \pm 54.2(149-298)$ & $251.4 \pm 53.0(163-337)$ & $204.0 \pm 30.0(147-255)$ & $246.6 \pm 34.6(201-292)$ \\
$\mathrm{RBC} *\left(\times 10^{12} / \mathrm{L}\right)$ & $4.4 \pm 0.1(4.22-4.61)$ & $4.5 \pm 0.4(4.12-5.17)$ & $4.5 \pm 0.4(3.85-5.02)$ & $4.3 \pm 0.2(3.79-4.58)$ \\
$\mathrm{Cr}(\mu \mathrm{mol} / \mathrm{L})$ & $56.7 \pm 6.6(46-66)$ & $57.8 \pm 4.1(52-64)$ & $73.8 \pm 7.5(63-89)$ & $70.0 \pm 7.7(64-88)$ \\
ALT $(\mathrm{U} / \mathrm{L})$ & $15.4 \pm 2.7(13-22)$ & $14.7 \pm 5.6(9-26)$ & $13.0 \pm 4.1(5-17)$ & $13.4 \pm 6.8(7-29)$ \\
AST $(\mathrm{U} / \mathrm{L})$ & $23.2 \pm 6.6(16-39)$ & $19.7 \pm 2.8(17-24)$ & $16.3 \pm 3.2(12-21)$ & $19.8 \pm 5.0(14-31)$ \\
ALB $(\mathrm{g} / \mathrm{L})$ & $44.0 \pm 2.5(39.3-48.3)$ & $44.2 \pm 2.3(41.7-48.8)$ & $43.1 \pm 2.9(38-46.8)$ & $43.2 \pm 2.4(39.9-48)$ \\
$\operatorname{TP}(\mathrm{g} / \mathrm{L})$ & $73.4 \pm 4.4(63.7-79)$ & $74.5 \pm 3.2(69.9-80.6)$ & $70.6 \pm 5.0(61.3-77.5)$ & $74.1 \pm 4.2(69.1-81.6)$ \\
TBIL $(\mu \mathrm{mol} / \mathrm{L})$ & $12.5 \pm 5.5(5.2-21.8)$ & $10.9 \pm 4.6(6.7-20)$ & $14.2 \pm 3.4(9.6-19.6)$ & $12.1 \pm 3.1(8-18)$ \\
DBIL $(\mu \mathrm{mol} / \mathrm{L})$ & $2.3 \pm 1.7(0.17-4.93)$ & $2.0 \pm 2.0(0.05-5.56)$ & $3.3 \pm 1.4(1.39-5.74)$ & $1.7 \pm 0.9(0.58-3.45)$ \\
\hline
\end{tabular}

Figures in parentheses indicate ranges. BMI, Body mass index; PLT*, platelet count; RBC*, red blood cell count; Cr, serum creatinine; ALT, alanine aminotransferase; AST, aspartate aminotransferase; ALB, albumin; TP, total protein; TBIL, total bilirubin; DBIL, direct bilirubin.

The fits for the observed value versus the PRED and the observed value versus the IPRED concentrations were judged to be good and the trend line was close to the line of unity, as shown in Figure 1A and 1B. The conditional weighted residuals (CWRES) value versus the PRED plots showed the points to be symmetrically distributed around a straight line through 0 , with most of the points being within -4 and +4 units, as shown in Figure 1C. The distribution of the CWRES fits to a nearly symmetrically distribution, as shown in Figure 1D. Therefore, there was no evidence of bias. Overall, the plots indicated that the study data were sufficiently well described by the final model that was developed in this study.

\section{Model validation}

Model validation is very important in population analyses ${ }^{[17]}$. In the present study, bootstrap and pcVPC analyses were used to evaluate model stability.

The results of the bootstrap method are shown in Table 2 . The median values of the PK parameter estimates obtained after 1000 runs, $100 \%$ of which were minimisation successful, were not significantly different from the estimates of the original dataset, and the $95 \%$ confidence interval was within reasonable limits.

The pcVPC results are presented in Figure 2. The plots showed that the observed plasma concentration data mostly fit well within the 5th-95th percentiles of the simulated data, except for the data of one subject in the infusion group.

\section{Population pharmacokinetic/pharmacodynamic modelling}

$\mathrm{ACT}$ was chosen as the PD endpoint because it is recommended that the ACT should be monitored to ensure the desired degree of anticoagulation and to minimise the risk of bleeding complications during bivalirudin treatment ${ }^{[18]}$.
Table 2. Parameter estimates of the final pharmacokinetic model and bootstrap validation.

\begin{tabular}{|c|c|c|c|c|}
\hline \multirow[t]{2}{*}{ Parameter } & \multicolumn{2}{|c|}{$\begin{array}{c}\text { Estimates of } \\
\text { the model parameters }\end{array}$} & \multirow[b]{2}{*}{ Median } & \multirow{2}{*}{$\begin{array}{c}\text { Bootstrap } \\
\text { 2.5th, 97.5th } \\
\text { Percentiles }\end{array}$} \\
\hline & Estimates & SE\% & & \\
\hline \multicolumn{5}{|c|}{ Pharmacokinetic parameter } \\
\hline $\mathrm{CL}\left(\mathrm{L} \cdot \mathrm{h}^{-1} \cdot \mathrm{kg}^{-1}\right)$ & 0.323 & 2.6 & 0.323 & $0.307,0.339$ \\
\hline$V_{1}(\mathrm{~L} / \mathrm{kg})$ & 0.086 & 4.2 & 0.0855 & $0.079,0.094$ \\
\hline $\mathrm{Q}\left(\mathrm{L} \cdot \mathrm{h}^{-1} \cdot \mathrm{kg}^{-1}\right)$ & 0.0957 & 3.0 & 0.0959 & $0.0908,0.102$ \\
\hline$V_{2}(\mathrm{~L} / \mathrm{kg})$ & 0.0554 & 3.3 & 0.0555 & $0.052,0.059$ \\
\hline \multicolumn{5}{|c|}{ Inter-individual variability } \\
\hline $\mathrm{CL}(\%)$ & 14.8 & 19.4 & 14.2 & $8.63,20.0$ \\
\hline$V_{1}(\%)$ & 24.2 & 29.1 & 23.3 & $8.18,36.2$ \\
\hline Q (\%) & $--^{a}$ & $-^{a}$ & $--^{a}$ & $-^{a}$ \\
\hline$V_{2}(\%)$ & 15.6 & 14.7 & 15.1 & $9.75,19.4$ \\
\hline \multicolumn{5}{|l|}{ Residual variability } \\
\hline Proportional error (\%) & 0.08 & 10.2 & 0.08 & $0.07,0.09$ \\
\hline
\end{tabular}

a Inter-individual variability of Q could not be determined.

\section{Model building}

The PD response was characterised by a sigmoid $E_{\max }$ model without the Hill coefficient for the base PK-PD model.

The exponential model was chosen as the inter-individual variability model. A proportional error model was shown to be sufficient to characterise the residual variability. Twenty covariates, including demographic factors and laboratory indicators, were analysed. Only one covariate, the red blood cell count $\left(\mathrm{RBC}^{*}\right)$, showed a significant effect on the $\mathrm{EC}_{50}$. The 

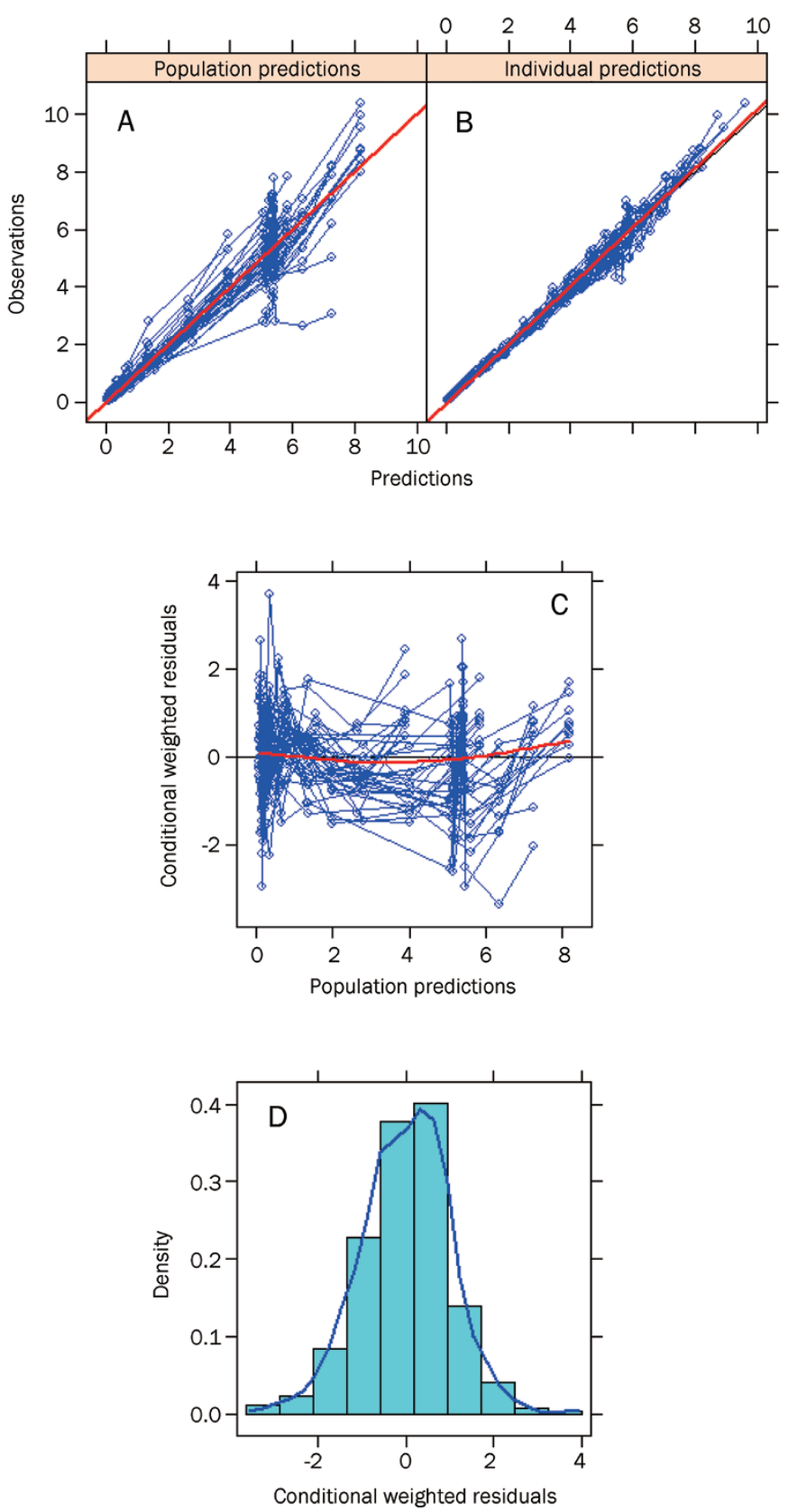

Figure 1. Goodness-of-fit plots of the final pharmacokinetic model. (A) Population predicted concentration versus observed concentration. (B) Individual predicted concentration versus observed concentration. (C) Conditional weighted residuals versus population predicted concentration. (D) Distribution of conditional weighted residuals. The solid line in (A) and (B) represent the line of unity, and that in (C) represents the line $y=0$.

OFV declined by $22.008(P<0.001)$ after the effect of RBC* on the $\mathrm{EC}_{50}$ was incorporated. Therefore, the covariate was retained in the final PK-PD model. The final covariate model was as follows:

$$
\left(\mathrm{EC}_{50}\right)_{\mathrm{i}}=\theta_{\mathrm{EC}_{50}}{ }^{*} \operatorname{Exp}\left(\eta_{\mathrm{EC}_{50}}\right)^{*}\left[1+\theta_{\mathrm{RBC}}\left(\mathrm{RBC}^{*}{ }_{\mathrm{i}}-4.40\right)\right]
$$

where $\theta_{\mathrm{RBC}}$ was the coefficient of the $\mathrm{RBC}^{*}$ effect on the $\mathrm{EC}_{50}$.

Figure $3 \mathrm{~B}$ demonstrated that the observed value versus the
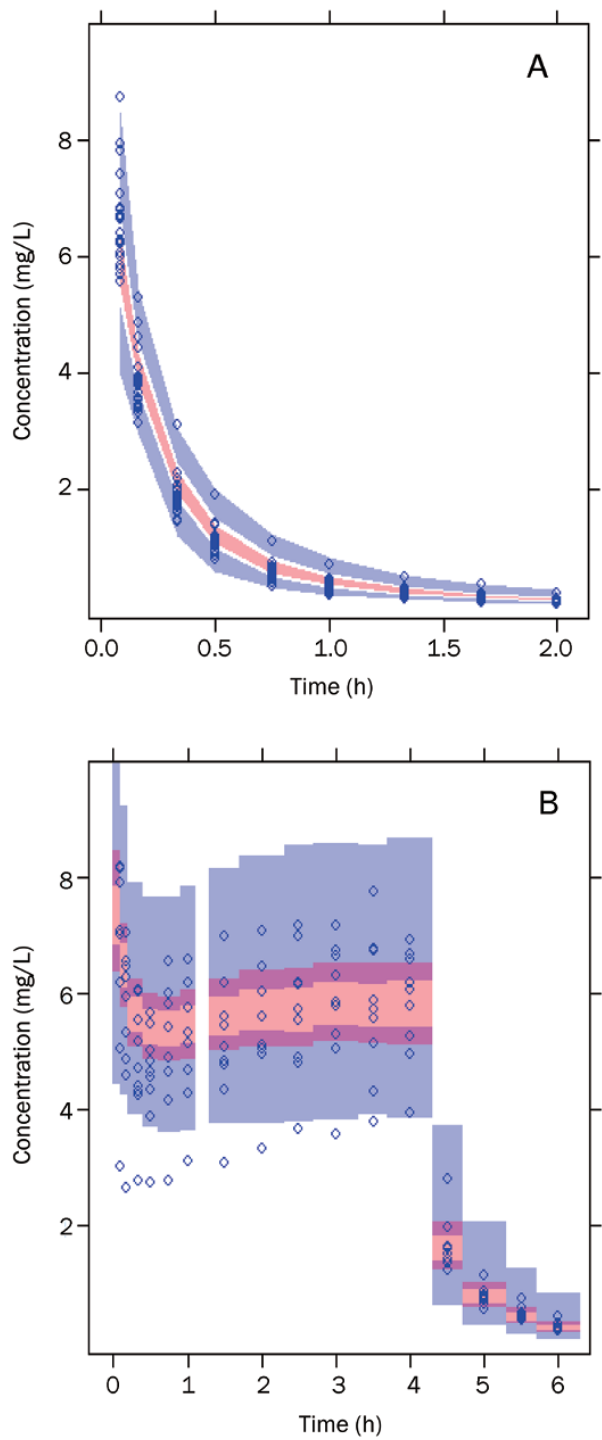

Figure 2. pcVPC results of the final pharmacokinetic model. The circles represent the observed data. The upper and bottom shaded areas correspond to the $90 \% \mathrm{Cl}$ of the 5th and 95th percentiles and the middle shaded areas correspond to the $90 \% \mathrm{Cl}$ of the median. (A) Three intravenous bolus injection groups. (B) The intravenous bolus injection group followed by infusion for $4 \mathrm{~h}$.

IPRED ACT tends to have good fitness. The CWRES value versus the PRED plots showed that the points were evenly distributed around a straight line through 0 , with most of them being within -3 and +3 units of the line, as shown in Figure 3C. The distribution of the CWRES shows nearly symmetrically distribution, as shown in Figure 3D. Therefore, there was no evidence of bias, indicating that the final PK-PD model described the data reasonably well.

\section{Model validation}

As for PK modelling described above, bootstrap and pcVPC analyses were used to evaluate the stability of the final PK-PD model. 

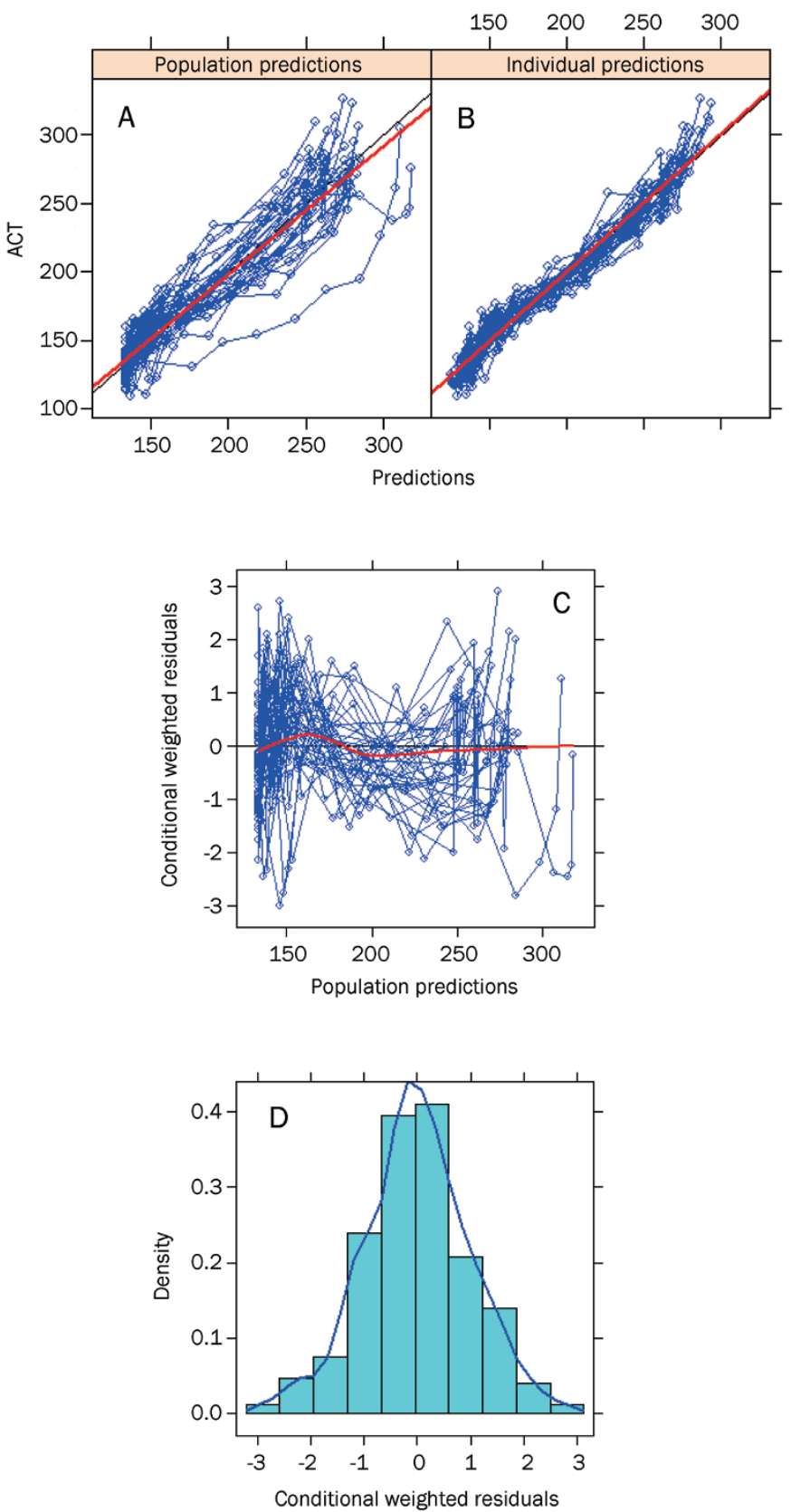

Figure 3. Goodness-of-fit plots of the final pharmacokinetic-pharmacodynamic model. (A) Population predicted activated clotting time (ACT) value versus observed ACT. (B) Individual predicted ACT versus observed value. (C) Conditional weighted residuals versus population predicted concentration. (D) Distribution of conditional weighted residuals. The solid line in (A) and $(B)$ represent the line of unity, and that in (C) represents the line $y=0$.

The results of the bootstrap method are shown in Table 3 . The median values of the PD parameter estimates obtained after 1000 runs, 992 of which were minimisation successful, were not significantly different from the estimates of the original dataset, and the $95 \%$ confidence interval was within reasonable limits. Furthermore, the $95 \%$ confidence interval of $\theta_{\mathrm{RBC}}$ did not include 0 , suggesting that the final population
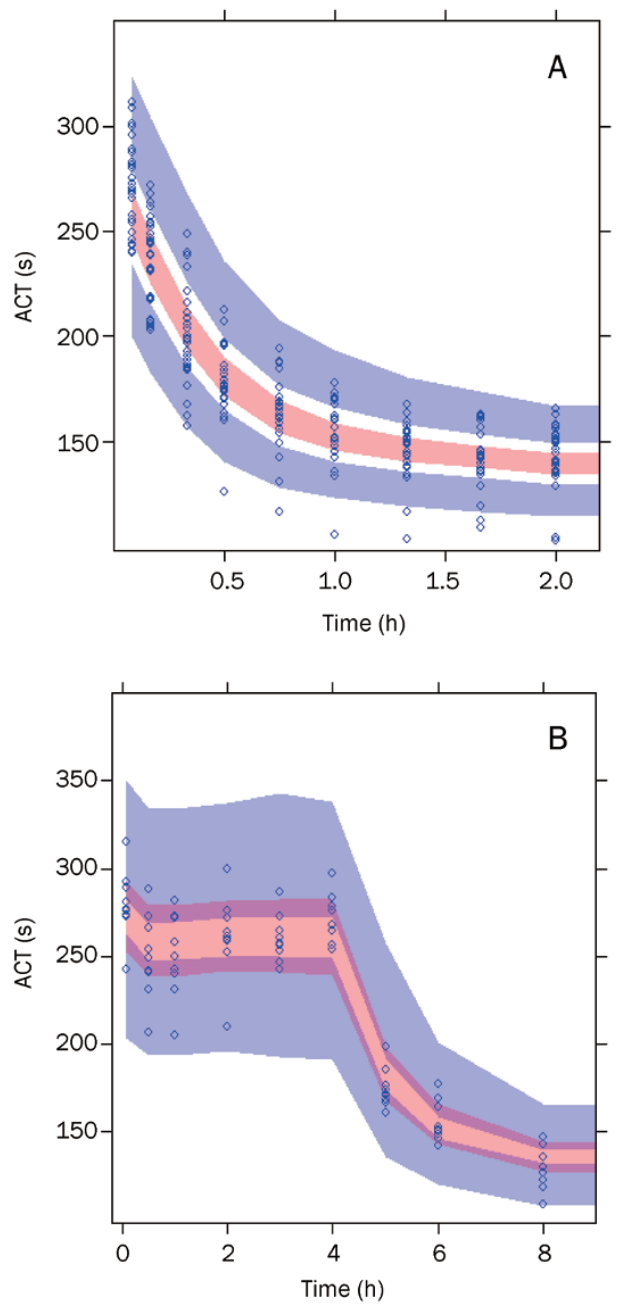

Figure 4. pcVPC results of the final pharmacokinetic-pharmacodanamic model. The circles represent the observed data. The upper and bottom shaded areas correspond to the $90 \%$ confidence interval $(\mathrm{Cl})$ of the 5th and 95th percentile. The middle shaded areas corresponded to the 90\% $\mathrm{Cl}$ of the median. (A) Three intravenous bolus injection groups. (B) The intravenous bolus injection followed by infusion for $4 \mathrm{~h}$ group.

PK-PD model was stable.

The pcVPC results are presented in Figure 4. The plots showed that the observed ACT value mostly fit well within the 5th-95th percentiles of the simulated data.

\section{Discussion}

In this study, we applied a population approach to evaluate the PK-PD characteristics of bivalirudin in healthy Chinese volunteers. To the best of our knowledge, this is the first attempt to identify a population PK-PD model for bivalirudin.

For PK model building, a two-compartment model was found to provide the best fit for the plasma concentration of bivalirudin. The mean population estimate for the clearance rate (CL) of bivalirudin in healthy Chinese subjects was similar to that calculated by a non-compartment method using WinNonlin, but the distribution volume of the two methods 
Table 3. Parameter estimates of the final pharmacokinetic-pharmacodynamic model and bootstrap validation.

\begin{tabular}{|c|c|c|c|c|}
\hline \multirow[t]{2}{*}{ Parameter } & \multicolumn{2}{|c|}{$\begin{array}{c}\text { Estimates of } \\
\text { the model parameters }\end{array}$} & \multirow[b]{2}{*}{ Median } & \multirow{2}{*}{$\begin{array}{l}\text { Bootstrap } \\
\text { 2.5th, 97.5th } \\
\text { Percentiles }\end{array}$} \\
\hline & Estimates & SE\% & & \\
\hline \multicolumn{5}{|c|}{ Pharmacodynamic parameter } \\
\hline$E_{\max }(\mathrm{s})$ & 318 & 2.39 & 320 & 304,340 \\
\hline $\mathrm{EC}_{50}(\mathrm{mg} / \mathrm{L})$ & 2.44 & 11.8 & 2.51 & $1.88,2.99$ \\
\hline$E_{0}(\mathrm{~s})$ & 134 & 0.98 & 134 & 131,136 \\
\hline$\theta_{\mathrm{RBC}}$ for $\mathrm{EC}_{50}$ & 1.70 & 3.54 & 1.69 & $0.23,1.73$ \\
\hline \multicolumn{5}{|c|}{ Inter-individual variability } \\
\hline$E_{\max }(\%)$ & 6.80 & 28.5 & 6.33 & $0.50,9.33$ \\
\hline $\mathrm{EC}_{50}(\%)$ & 46.4 & 28.6 & 27.1 & $0.45,62.6$ \\
\hline$E_{0}(\%)$ & 4.10 & 19.2 & 4.17 & $2.27,5.58$ \\
\hline \multicolumn{5}{|l|}{ Residual variability } \\
\hline Proportional error, \% & 4.67 & 17.0 & 4.56 & $2.45,6.06$ \\
\hline
\end{tabular}

$\theta_{\mathrm{RBC}}$ was the coefficient of the RBC* on $\mathrm{EC}_{50}$.

was somewhat different ${ }^{[13]}$. This result may be because the $\mathrm{CL}$ is mainly determined by the hepatic or renal function of the subject and is less dependent on the parameter calculation method. Meanwhile, the distribution volume is a hypothetical parameter used to evaluate the extent of distribution of a drug. In other words, the distribution volume does not have any real meaning in physiology, and may differ when calculated by either the non-compartment or the compartment method.

The goal of building the covariate model was to find subjectspecific characteristics, which could explain, and thus reduce, the variability of the model. We tested the effect of 30 covariates, including age, weight, gender, creatinine, alanine aminotransferase (ALT), albumin (ALB), etc on PK parameters. However, no covariate showed a significant effect on the PK model. This result may be due to two reasons, the first being the PK characteristics of bivalirudin. Bivalirudin is eliminated via the dual pathways of proteolysis and renal excretion, avoiding the hepatic metabolism pathways, which may correlate with several biological characteristics, such as ALT, ALB, etc. Additionally, bivalirudin was administered intravenously. This avoided the first-pass metabolism that may be influenced by some covariates. In this study, the bivalirudin dose was administered by weight adjustment, which may neutralise the effect of weight on PK parameters. The second reason that there was not a significant covariate effect in the PK model may be due to the nature of the subjects enrolled in the study. This was a phase I study conducted on healthy subjects, which was a uniform population with a relatively narrow range of covariates. For example, it was reported ${ }^{[19,20]}$ that compared to the normal renal function (glomerular filtration rate, GFR $\geq 90$ $\mathrm{mL} / \mathrm{min}$ ) group, the $\mathrm{CL}$ of bivalirudin was unchanged in mild renal impairment (GFR $60-89 \mathrm{~mL} / \mathrm{min}$ ), and decreased by $45 \%$ and $68 \%$ in moderate (GFR $30-59 \mathrm{~mL} / \mathrm{min}$ ) and severe
(GFR $<30 \mathrm{~mL} / \mathrm{min}$ ) renal impairment, respectively. However, this result could not be demonstrated with the data at han, because all 36 subjects enrolled in this study had normal renal function (GFR $>90 \mathrm{~mL} / \mathrm{min}$ ).

For PK-PD model building based on the anticoagulation mechanism of bivalirudin, a direct response, sigmoid $E_{\max }$ effect model without the Hill coefficient factor was chosen as the basic model. We tested 20 covariates, and only the RBC* showed a significant effect on the $\mathrm{EC}_{50}$. The biological basis for this phenomenon is currently unknown; however, a preliminary hypothesis may be offered. For the ACT measurements, 3-mL blood samples were collected in a cuvette containing a number of dried reagents, including phospholipid, silica, kaolin, stabilisers, and buffers, and the ACT determination was performed immediately. If the blood samples were diluted, the concentration of the blood coagulation factors would decrease, leading to a prolonged ACT value. The low $\mathrm{RBC}^{*}$ condition is somewhat similar to that of diluted blood; therefore, it will have a lower $\mathrm{EC}_{50}$. The observed effect of the $\mathrm{RBC}^{*}$ on the $\mathrm{EC}_{50}$ may be due to the relatively low concentrations of blood coagulation factors that were present in the low $\mathrm{RBC}^{*}$ condition, rather than the "real" effect of the RBC. However, the exact mechanism needs further investigation.

For modelling diagnostics and validation, the CWRES and the pcVPC methods were used in this study instead of the traditionally used WRES and VPC methods. The CWRES and pcVPC methods have been proposed recently ${ }^{[15,21]}$. Use of the WRES as a diagnostic tool when performing modelling using the FOCE methods leads to the possibility of misguided model development and diagnosis, or, at the very least, less informed model development. The CWRES, on the other hand, are calculated similarly to the WRES, but are based on the FOCE approximation. The FOCE method was used for building the model used in this study. Therefore, the CRWES was more appropriate than the WRES for diagnostics of the established models. The principal benefit of the pcVPC method has been explored by application to both simulated and real examples of PK and PK-PD models ${ }^{[12]}$. To enhance the ability to diagnose model misspecifications, we used the pcVPC method instead of the traditional VPC method.

Generally speaking, the developed PK and PK-PD models fit the observed data well, despite some underestimations when ACT values were high in the PK-PD model and the fact that there was a one outlier subject in the pcVPC of the infusion group. The demographic and biological characteristics of the subject were all within the normal ranges. Furthermore, the records of both the drug administration and the sampling process regarding this subject were checked again and no mistakes had been made. As mentioned above, bivalirudin is not metabolised via cytochrome P450s (CYPs) or drug transporters. Therefore, the reason for this outlier could not be explained by a gene polymorphism, and the definitive reason for the presence of this outlier is unclear.

This study has several limitations. First, the models are based on data from a total of 36 subjects, which may be considered a small number for population analysis. Second, all 
the subjects are healthy volunteers, with a narrow distribution of demographic and biological variables. For example, the average age was 33 years and the standard deviation was only 2 years. There were no subjects older than 37 years, only three subjects were 29 years, and all of the others were between 30 and 37 years old. This limits the use of this model to predict the PK and PD of bivalirudin in the elderly. In addition, the accuracy of any prospective simulations using parameter estimates and their variability may be impaired if applied to patients using bivalirudin because the subjects involved in the model-building process were all healthy volunteers. The stability and extrapolation of the models developed in this study need further improvement and evaluation in a larger group of subjects, including patients, before the models can be fully validated.

In conclusion, the population PK and PK-PD models of bivalirudin in healthy, Chinese subjects were well developed. The base models of PK and PK-PD were a two-compartment model and a sigmoid $E_{\max }$ model without the Hill coefficient, respectively. No covariate was retained in the final PK model and one covariate, the $\mathrm{RBC}^{*}$, was incorporated into the final PK-PD model. These models provide a first step for using the population PK-PD study method in a phase I study of bivalirudin. These models may also help in the further development of bivalirudin.

\section{Acknowledgements}

This study was financially supported by Shenzhen Main Luck Pharmaceuticals, Inc. The authors would like to thank Jing-kai GU, Min LU, Ling-yue MA, Xiao LIU, Shuo-han TIAN, Xiaoyan SHENG, and Jing-zi LI for their assistance in performing the trial, and Yuan-cheng CHEN for his assistance in data analysis.

\section{Author contribution}

Yi-min CUI and Xia ZHAO designed the trial; Dong-mei ZHANG performed the trial and wrote the paper; Kun WANG and Qing-shan ZHENG analysed data, built the models and prepared the figures; Xia ZHAO and Zi-ning WANG performed the trial; and Yun-fei LI analysed data.

\section{References}

1 Kimmel SE, Sekeres M, Berlin JA, Ellison N. Mortality and adverse events after protamine administration in patientsundergoing cardiopulmonary bypass. Anesth Analg 2002; 94: 1402-8.

2 Toschi V, Lettino M, Gallo R, Badimon JJ, Chesebro JH. Biochemistry and biology of hirudin. Coron Artery Dis 1996; 7: 420-8.

3 Maraganore JM, Bourdon P, Jablonski J, Ramachandran KL, Fenton JW 2nd. Design and characterization of hirulogs: a novelclass of bivalent peptide inhibitors of thrombin. Biochemistry 1990; 29: 7095-101.
4 Vasquez JC, Vichiendilokkul A, Mahmood S, Baciewicz FA Jr. Anticoagulation with bivalirudin during cardiopulmonary bypass in cardiac surgery. Ann Thorac Surg 2002; 74: 2177-9.

5 Stone GW, McLaurin BT, Cox DA, Bertrand ME, Lincoff AM, Moses JW, et al. Bivalirudin for patients with acute coronary syndromes. N Engl J Med 2006; 355: 2203-16.

6 Lincoff AM, Bittl JA, Harrington RA, Feit F, Kleiman NS, Jackman $J \mathrm{D}$, et al. Bivalirudin and provisional glycoprotein IIb/IIla blockade compared with heparin and planned glycoprotein IIb/IIla blockade during percutaneous coronary intervention: REPLACE- 2 randomized trial. JAMA 2003; 289: 853-63.

7 Kastrati A, Neumann FJ, Mehilli J, Byrne RA, lijima R, Büttner HJ, et al. Bivalirudin versus unfractionated heparin during percutaneous coronary intervention. N Engl J Med 2008; 359: 688-96.

8 Stone GW, Witzenbichler B, Guagliumi G, Peruga JZ, Brodie BR, Dudek $\mathrm{D}$, et al. Bivalirudin during primary $\mathrm{PCl}$ in acute myocardial infarction. N Engl J Med 2008; 358: 2218-30.

9 Atkinson AJ Jr, Lalonde RL. Introduction of quantitative methods in pharmacology and clinical pharmacology: a historical overview. Clin Pharmacol Ther 2007; 82: 3-6.

10 Gobburu VS, Marroum PJ. Utilisation of pharmacokinetic-pharmacodynamic modelling and simulation in regulatory decision making. Clin Pharmacokinet 2001; 40: 883-92.

11 Grasela TH, Dement CW, Kolterman OG, Fineman MS, Grasela DM, Honig P. Pharmacometrics and the transition to model-based development. Clin Pharmacol Ther 2007; 82: 137-42.

12 Zhang LP, Pfister M, Meibohm B. Concepts and challenges in quantitative pharmacology and model-based drug development. AAPS J 2008; 10: 552-9.

13 Zhang DM, Wang ZN, Zhao X, Lu W, Gu JK, Cui YM. Pharmacokinetics, pharmacodynamics, tolerability and safety of single doses of bivalirudin in healthy Chinese subjects. Biol Pharm Bull 2011; 34: 1841-8.

14 Karlsson MO, Holford N. A tutorial on visual predictive checks. PAGE 2008; 17: Abstr 1434 [www.page-meeting.org/?abstract=1434].

15 Bergstrand M, Hooker AC, Wallin JE, Karlsson MO. Predictioncorrected visual predictive checks for diagnosing nonlinear mixedeffects models. AAPS J 2011; 13: 143-51.

16 Zhang L, Beal SL, Sheiner LB. Simultaneous vs sequential analysis for population PK/PD data I: best-case performance. J Pharmacokinet Pharmacodyn 2003; 30: 387-404.

17 Brendel K, Dartois C, Comets E, Lemenuel-Diot A, Laveille C, Tranchand B. Are population pharmacokineticand/or pharmacodynamic modelsadequately evaluated? A survey of the literaturefrom 2002 to 2004. Clin Pharmacokinet 2007; 46: 221-34.

18 The Medicines Company. BivalirudinPrescribing Information (2005).

19 Robson R. The use of bivalirudin in patients with renal impairment. J Invasive Cardiol 2000; 12: 33F-6.

20 Robson R, White H, Aylward P, Frampton C. Bivalirudin pharmacokinetics andpharmacodynamics: Effect of renalfunction, dose, and gender. Clin Pharmacol Ther 2002; 71: 433-9.

21 Hooker AC, Staatz CE, Karlsson MO. Conditional weighted residuals (CWRES): a model diagnostic for the FOCE method. Pharm Res 2007; 24: 2187-97. 